\title{
Photoluminescence Spectroscopy of the Molecular Biexciton in Vertically Stacked Quantum Dot Pairs
}

\author{
M. Scheibner I. V. Ponomarev, E. A. Stinaff, M. F. Doty, A. \\ S. Bracker, C. S. Hellberg, T. L. Reinecke, and D. Gammon \\ Naval Research Laboratory, Washington, DC 20375, USA
}

(Dated: October 31, 2018)

\begin{abstract}
We present photoluminescence studies of the molecular neutral biexciton-exciton spectra of individual vertically stacked InAs/GaAs quantum dot pairs. We tune either the hole or the electron levels of the two dots into tunneling resonances. The spectra are described well within a few-level, few-particle molecular model. Their properties can be modified broadly by an electric field and by structural design, which makes them highly attractive for controlling non-linear optical properties.
\end{abstract}

PACS numbers: 78.67.Hc, 73.21.La, 78.55.Cr

Quantum dots (QDs) provide an attractive active medium for nanophotonics, quantum optics and quantum information because they can be integrated into optoelectronic architectures, they interact strongly with light, and their properties are adjustable by growth. This functionality can be enhanced by building molecules of QDs (QDMs), in which new inter-dot transitions arise that are strongly tuned with electric field. Here we employ single, custom designed "diatomic" QDMs in order to explore the electric field response of a new type of optical excitation in QDMs - the molecular biexciton.

The exciton and biexciton states are at the heart of many quantum and nonlinear optical processes in a single QD [1]-[5]. Their energy level diagram is the starting point in all such studies. The energy of the biexciton is not exactly twice that of the exciton because of Coulomb and spin interactions. In a QDM these interactions again play a central role in the energy spectrum, but now intricately combined with coherent tunneling between dots. The resulting molecular biexciton spectra are very rich and sensitive to electric field, but their characteristic spectral pattern can be understood quantitatively using a relatively simple energy level diagram. The spectral patterns presented here are qualitatively similar for all QDMs that we have studied as long as we account for whether it is the electrons or the holes that tunnel between dots. We demonstrate both cases here. In this paper we consider neutral QDMs but a similar analysis can be applied to charged QDMs.

The QDM easiest to fabricate, and the one now under intense study, is the asymmetric diatomic molecule formed out of two non-identical InAs dots vertically stacked and separated by a thin GaAs tunnel barrier. In this study we used QDMs with tunnel barriers of $d=6$ $\mathrm{nm}$ and $d=14 \mathrm{~nm}$ (center to center $\tilde{d}=8.5 \mathrm{~nm}$ and 17 $\mathrm{nm})$ for hole and electron tunneling respectively. They were embedded in Schottky diodes. We chose the heights of the QDs such that the top QD had higher transition energy. Here we consider only the lower energy QD transition - the other is at substantially higher energy and plays no role. Further details on the growth and experi- mental methods can be found in Refs. [6, 7].

A typical optical spectrum of the bottom dot transitions in a single QDM exhibits a series of PL-line doublets (Fig. 1(a)). The lower energy lines in the doublets form a sequence of charged excitonic transitions, which is similar to that measured in single dots; i.e. $X^{-}\left(\frac{20}{10}\right)$, $X X\left(\underline{2}_{\underline{2}}^{0}\right), X\left(\underline{1}_{10}^{0}\right)$, and $X^{+}\left(\underline{1}_{\underline{2}}^{0}\right)$. Here the upper two numbers are the numbers of the electrons in the bottom and the top dot, respectively, and the lower two numbers are the numbers of holes in the two dots. The underlines denote the position of the recombining particles (in this case, identifying an intra-dot transition). The shifts in

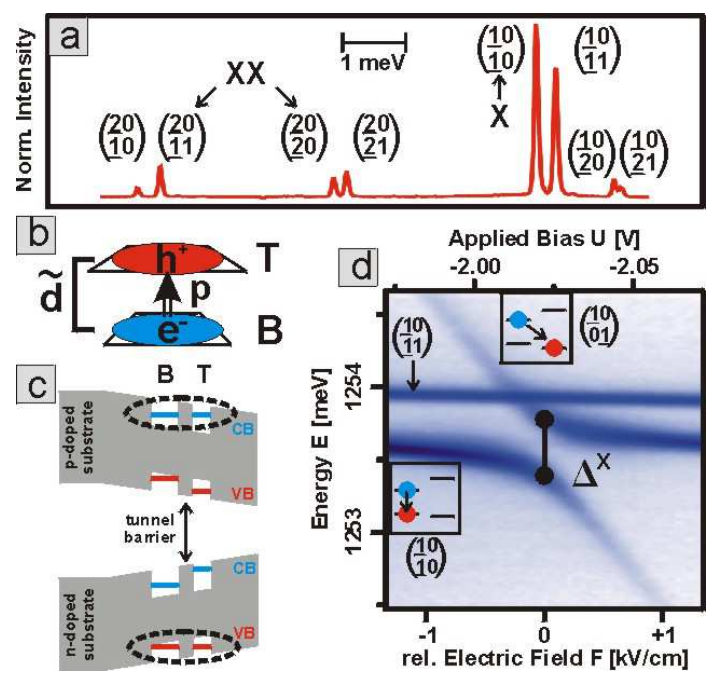

FIG. 1: (Color online) (a) PL spectrum of a QDM showing bottom dot intra-dot transitions $(U=-1.60 \mathrm{~V})$. (b) A large dipole moment $p \propto e \tilde{d}$ exists between the electron and hole, each located in separate QDs. (c) Electric field dependent PL spectrum at the hole level resonance of the neutral exciton obtained from a QDM with $\tilde{d}=8.5 \mathrm{~nm}$. An AC is seen at the resonance of intra-dot $\left(\frac{10}{10}\right)$ and inter-dot $\left(\frac{10}{01}\right)$ transition, arising from the formation of bonding and antibonding molecular hole states with a splitting of $\Delta^{X}=420$ $\mu \mathrm{eV}$. (d)QDM diode structures for electron (top) and hole (bottom) level resonances (B: bottom QD, T: top QD). 
the energies between the differently charged exciton transitions are well known in the spectra of single dots and arise from quantitative differences in the Coulomb interactions of the holes and electrons. The second line in each doublet is a new transition characteristic of a QDM. It is a Stark shifted replica of the first line, caused by the electric field of an extra charge in the top dot (here one hole) [8]. Note in particular the new $X^{-}$-like biexciton transition $\left(\frac{20}{11}\right)$. These assignments are solidified below. This energy structure serves as a useful basis for the interpretation of the full and often complex molecular spectrum.

A QDM allows for two types of optical transitions - (i) the intra-dot transition mentioned above in which only one QD is involved, and (ii) the inter-dot transition in which different QDs are involved. For the intra-dot exciton transition, as for single dots, the static dipole moment (experimentally measured by the shift of the transition energy with electric field) is fairly small. In contrast, for the inter-dot transition the static dipole moment is very large because of the separation, $\tilde{d}$, between the dots ( $p \propto e \tilde{d}$ where $e$ is the electron charge) (Fig. 1(b)).

The molecular nature of these QD pairs is revealed in the coherent superposition of states that arises when the electric field brings the intra-dot and inter-dot exciton states energetically into resonance. Because the two dots in the QDM have different transition energies, either the electron or the hole (within the exciton) can tunnel, but not both (Fig. 1(c)) 7]. This has been shown for a single exciton in neutral and in charged QDMs 9]-[12] (see also Fig. 3). We consider first the case in which the hole levels of the two dots are near resonance, and the two electron levels are detuned far from resonance. Coherent tunneling of holes at these resonances leads to an anticrossing (AC) in the energy levels and to a mixing of the properties of the exciton states. For the exciton $(X)$, a single AC of energy $\Delta^{X}=430 \mu \mathrm{eV}$ is seen in the PL spectrum (Fig. 1(d)).

If two electron-hole pairs are excited in the system, a biexciton $(X X)$ is formed. The biexciton state allows a (conditional) two photon cascade - optical transitions from the biexciton state to the exciton states, and from the exciton states to the ground state (exciton vacuum). In a single dot, the lowest energy state of the biexciton is a spin singlet with two electrons and two holes, each spin-paired in s-shell orbitals. As one might expect, the quantum states of a molecular biexciton are significantly richer than in a single QD because the carriers can be distributed over both dots. Nevertheless, as we now show, the regular pattern found experimentally, and from analysis, leads to a simple, intuitive understanding of the molecular biexciton.

Figure 2(a) shows the calculated energies for the biexciton $(X X)$ and the exciton $(X)[13,14]$. The biexciton can take several configurations of the two electrons and two holes over the two dots. In the hole-resonance case, both electrons relax to the s-shell orbital of the low en-

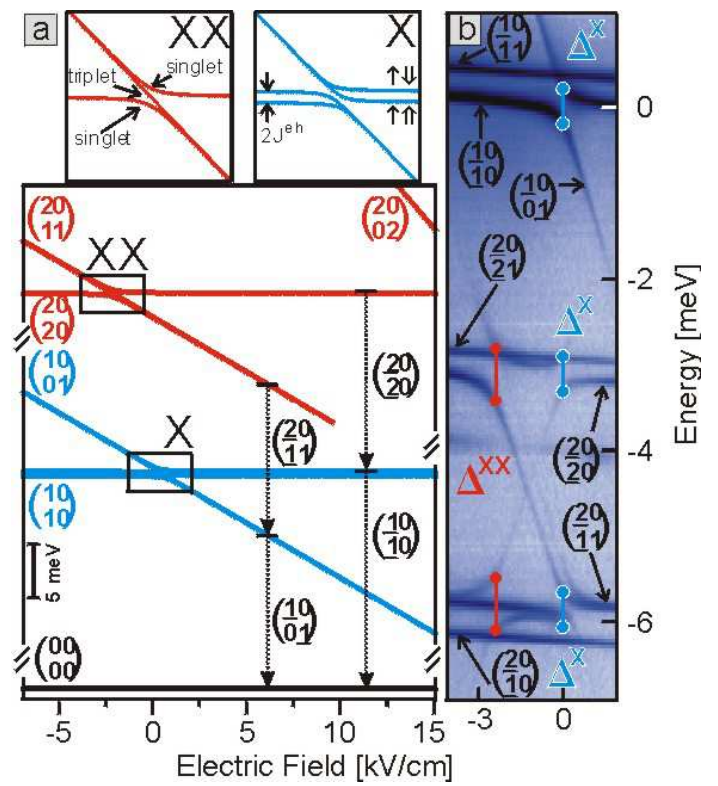

FIG. 2: (Color online) (a) The calculated energy level diagram of the neutral biexciton-exciton cascade [13, 14]. The areas in the boxes $X$ and $X X$ have been enlarged to show the fine structure caused by spin exchange interactions in the vicinity of level resonances. (b) Extended electric field dependent optical spectrum of the QDM (same as in Fig. 1(d)) with neutral exciton $(X)$ and neutral biexciton $(X X)$ transitions.

ergy dot, and thus form an electron spin singlet configuration by the Pauli Principle. The two holes can be in either of the two dots. We calculate energies relative to that configuration in which all particles are in the low energy dot. Thus, with all electrons and holes in the same dot, $\left(\begin{array}{c}20 \\ 20\end{array}\right)$, the slope as a function of electric field is zero. With only one hole in the other dot, $\left(\begin{array}{l}20 \\ 11\end{array}\right)$, the slope is $p$. With two holes in the other $\operatorname{dot}\left(\begin{array}{l}20 \\ 02\end{array}\right)$, the slope is $2 p$. For the configuration in which the two holes are separated from the two electrons $\left(\begin{array}{c}20 \\ 02\end{array}\right)$, there is a large Coulomb interaction that substantially increases its energy.

Molecular features in the biexciton spectrum appear along with the exciton spectrum in Fig. 2(b), where we present a new view of the data of Fig. 1(d) over a wider energy range. There are several observations that allow us to identify, and understand this spectrum and to define fitting parameters that can largely be found independently from each other and need to be fine tuned only for quantitative agreement.

(i) There is an "X-pattern" that ranges between two extremes in energy, that are determined by the biexciton intra-dot transitions in Fig. 2(b). On the high energy side, the "X-pattern" is bounded by a nearly horizontal line that corresponds closely to the previously known biexciton transition energy of a single dot. Thus, we were able to conclude that this is the intra-dot transition $\left(\frac{2}{20}\right)$. On the low energy side of the "X-pattern", the transition is $X^{-}$-like, which means, close to the transition energy 
of a negative exciton in the bottom $\operatorname{dot}\left(\frac{20}{10}\right)$. Thus, we deduce that this low energy line is the intra-dot transition $\left(\frac{20}{1} 1\right)$. As mentioned above, it is shifted from the negative exciton transition because of the presence of a second hole in the other dot.

(ii) Within the "X-pattern" there are two inter-dot transitions that anticross with the two intra-dot transitions. The "X-pattern" observed in the data occurs because the biexciton and exciton states each have energy ACs that are close in electric field (because of the relatively small differences in Coulomb energies for the states involved). One pair of these AC resonances occurs at precisely the same electric field as the single exciton resonance. This electric field alignment happens because the biexciton makes a transition to the exciton state, and thus the $\mathrm{AC}$ of the exciton state appears in the optical transitions of both the exciton and biexciton. Consequently, the ACs on the left side of the biexciton spectrum in Fig. 2(b) must arise from the biexciton level ACs. The AC energy for the biexciton levels is $\Delta^{X X}=630 \mu \mathrm{eV}$ $\approx \sqrt{2} \Delta^{X}$ because two indistinguishable holes can tunnel [11].

(iii) The distinctive fine structure splittings in the biexciton spectrum, which are best seen at the two energetically lowest ACs in Fig. 2(b), arise from spin. The biexciton states in which both holes are in the same dot, $\left(\begin{array}{l}20 \\ 20\end{array}\right)$ and $\left(\begin{array}{l}20 \\ 02\end{array}\right)$, are spin singlets. If the two holes are each in a separate dot, $\left(\begin{array}{l}20 \\ 11\end{array}\right)$, the biexciton can exist in a spin singlet or in one of three spin triplet states. Because tunneling conserves spin, singlet states anticross only with singlet states. In essence, Pauli blocking prevents the triplets from tunnel-coupling with a singlet. Thus, the triplet states pass straight through the biexciton AC, and there is a "kinetic exchange splitting" of the singlet and triplet state energies that arises from tunneling and Pauli blocking (top left inset of Fig. 2(a)). Moreover, the additional fine structure splitting in the exciton [6], arising from exchange of the unpaired electron and hole $\left(J^{e h}\right)$ (top right inset of Fig. 2(a)), is seen clearly in the transition spectrum between biexciton and exciton. This fine structure does not appear in optical transitions from the exciton state because one transition is optically dark. These exchange splittings in the calculated energy diagrams (Fig. 2(a)) can be traced to the corresponding fine structure patterns observed in the measured spectrum (Fig. 2(b)). Thus, we find that our understanding of the fairly complex spectral pattern of the biexciton is described remarkably very well by the simple energy level diagrams of Fig. 2(a).

We can also engineer the asymmetric QDM structure to induce the electron to tunnel instead of the holes [7]. In Fig. 3 we compare the two cases of hole and electron tunneling over a large bias range. We focus on the biexciton-exciton cascade spectra in the solid red boxes. The same discussion given above for the hole resonance applies - with two major differences: (i) Because elec-

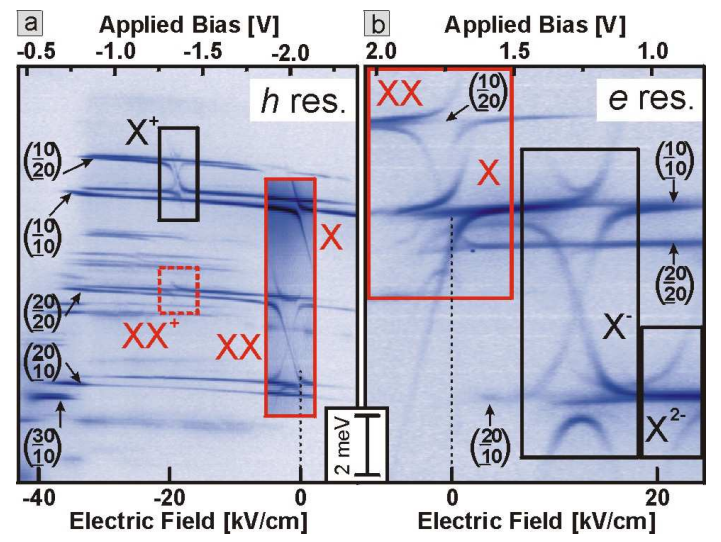

FIG. 3: (Color online) (a) Hole and (b) electron level resonances in the field dependent PL spectra of a QDM. The red boxes highlight the regions were the molecular resonances of exciton, biexciton and charged biexciton are seen. The area in the solid red box in (a) was taken with 5 times higher resolution in the field direction and 8 times longer integration time. The black boxes outline the patterns of $X^{+}, X^{-}$and $X^{2-}$, which were previously studied [6, 11, 12]. The spectra are centered at (a) $1251.57 \mathrm{meV}$ and (b) $1297.21 \mathrm{meV}$.

trons instead of holes now occupy the top QD in the inter-dot configurations, e.g. $\left(\begin{array}{l}01 \\ 10\end{array}\right)$ instead of $\left(\begin{array}{l}10 \\ 01\end{array}\right)$, the sign of the inter-dot dipole moment is reversed. (ii) The biexciton "X-pattern" spectrum now overlaps the exciton spectrum; in contrast to the hole resonance case, where it was shifted well below in energy. This difference arises because of the different nature of one of the direct transitions that bounds the "X-pattern". In particular, the $(\underline{11} 0)$ direct transition that occurs in the electron resonance case is now shifted above the neutral exciton energy and close to the energy of a positive exciton $\left(X^{+}-\right.$ like). This is in contrast to the corresponding transition for the hole resonance case, $\left(\frac{20}{11}\right)$, whose energy was close to the negative exciton $\left(X^{-}\right.$-like) and at an energy much lower than the exciton. For illustration, we compare cal-

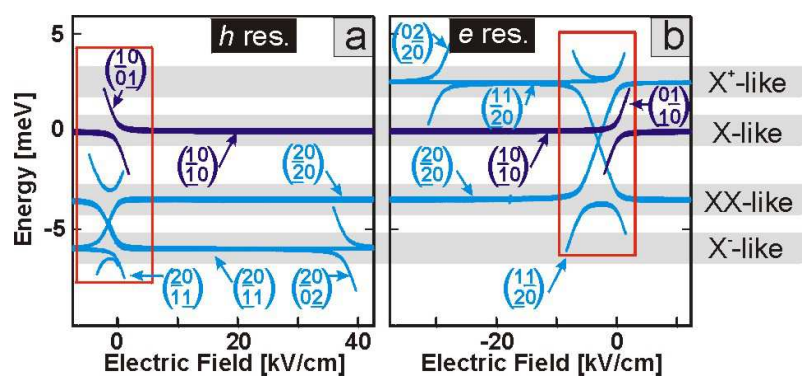

FIG. 4: (Color online) Calculated optical spectra of the neutral excitons (blue) and the corresponding biexcitons (cyan) if (a) the hole levels and (b) the electron levels are tuned into resonance. For illustration we have arbitrarily taken the same parameters for both cases - in meV: $E^{e h}=17.5, E_{1}^{h}=14.8$, $E_{1}^{e}=23.3, J^{e h}=0.2, \Delta^{X}=1.0, p=\frac{ \pm 1}{(\mathrm{kV} / \mathrm{cm})}[13,14,15]$. 


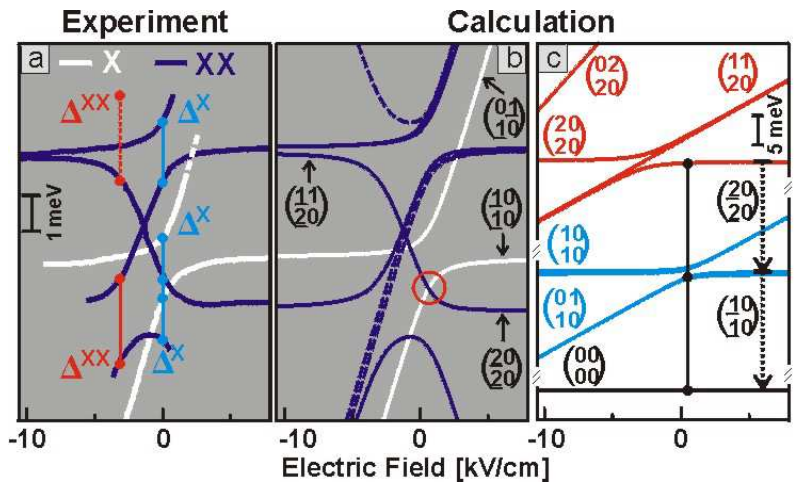

FIG. 5: (Color online) (a) Measured field dependent transition energies of the neutral biexciton cascade from the red boxed region in Fig. 3 (b) ( $X$ (white) and $X X$ (blue) PLlines). (b) Fitted PL-spectrum [13, 15]. (c) The corresponding level diagram.

culated transition spectra for the two cases in Fig. [4 The boxed regions reproduce qualitatively the measured spectra (red boxes in Fig. 3i) very well.

For a more quantitative analysis of the electron tunneling case we focus on the area in the red box in Fig. 3(b) (see Figs. [5(a) and (b)). Because the electron mass is much smaller than the hole mass, the $\mathrm{AC}$ energy is much larger $(1.65 \mathrm{meV})$ than for the hole resonance case $(0.45 \mathrm{meV})$ shown above, even with a dot separation twice as large. This leads to other quantitative differences in the spectrum as well. In particular, relative intensities are significantly changed because of more efficient thermalization and differences in oscillator strengths, which prevent the observation of some lines (dashed in Fig. 5(b)).

The molecular cascade transitions that are similar to those for a single dot, $\left(\frac{20}{20}\right)$ and $\left(\frac{10}{10}\right)$, are shown in Fig. [5 (c) by the dashed vertical arrows at $F=6 \mathrm{kV} / \mathrm{cm}[13$, 15]. With QDMs the transition energies are strongly tunable with bias. For example, moving to the left from $F=6$ $\mathrm{kV} / \mathrm{cm}$ in Fig. 5 (c), the exciton and biexciton transition energies change continuously, reaching a field where the two become equal [5]. This point is shown by the solid vertical lines in Fig 5 (c), and corresponds to the circled crossing point in Fig. 5(b) at $F=0.6 \mathrm{kV} / \mathrm{cm}$. Such resonances are realized for electron as well as hole tunneling. For this case the molecular design results in significant oscillator strength for both cascade transitions. In both cases we obtain an excellent fit of the measured biexciton-exciton spectrum, as seen by the comparison of Figs. 5 (a) and (b). Thus we find a common qualitative understanding of both types of neutral biexciton/exciton spectra in asymmetric QDMs.

We have presented the two-photon cascade spectra in QDMs and shown that their energy levels are widely adjustable by structural design and with electric field. Fi- nally we note that the biexciton and exciton in an uncharged QDM is only one example of this class of transitions. We have also observed spectra for singly (see dashed box Fig. 3(a)) and doubly charged QDMs, in which cases the transitions take place in the presence of resident carriers. Extension of the current work to these cases will provide the added opportunities of using longlifetime spin based quantum memories, and entanglement between the photons and the resident carriers.

We acknowledge the financial support by NSA/ARO and ONR. E.A.S., I.V.P., and M.F.D. thank the NRC/NRL for financial support.

* Electronic address: scheibner@bloch.nrl.navy.mil

[1] C. Santori, et al., Phys. Rev. B 66, 045308 (2002).

[2] R. M. Stevenson, et al., Nature 439, 179 (2006).

[3] N. Akopian, et al., Phys. Rev. Lett. 96, 130501 (2006).

[4] X. Li, et al., Science 301, 809 (2003).

[5] M. E. Reimer, et al., arXiv:0706.1075v1 (2007).

[6] M. Scheibner, et al., Phys. Rev. B 75, 245318 (2007).

[7] A. S. Bracker, et al., Appl. Phys. Lett. 89, 233110 (2006).

[8] At high excitation powers also triplets of PL-lines can be seen, corresponding to 0,1 , and 2 charges in the top QD.

[9] H. J. Krenner, et al., Phys. Rev. Lett. 94, 057402 (2005).

[10] G. Ortner, et al., Phys. Rev. Lett. 94, 157401 (2005).

[11] E. A. Stinaff, et al., Science 311, 636 (2006).

[12] H. J. Krenner, et al., Phys. Rev. Lett. 97, 076403 (2006).

[13] For hole or electron tunneling the molecular neutral exciton energies are found from the Hamiltonian with the basis spin states consisting of two bright, $\left(\begin{array}{l}\uparrow 0 \\ \Downarrow 0\end{array}\right),\left(\begin{array}{l}\uparrow 0 \\ 0 \Downarrow\end{array}\right)$ or $\left(\begin{array}{c}0 \uparrow \\ \Downarrow 0\end{array}\right)$, and two dark, $\left(\begin{array}{c}\uparrow 0 \\ \Uparrow 0\end{array}\right),\left(\begin{array}{c}\uparrow 0 \\ 0 \Uparrow\end{array}\right)$ or $\left(\begin{array}{c}0 \uparrow \\ \Uparrow 0\end{array}\right)$, excitons

$$
\widehat{H}_{X}=\widehat{I} \varepsilon_{X}+\left(\begin{array}{cccc}
J^{e h} & \frac{1}{2} \Delta^{X} & 0 & 0 \\
\frac{1}{2} \Delta^{X} & E^{e^{h}}+p F & 0 & 0 \\
0 & 0 & -J^{e h} & \frac{1}{2} \Delta^{X} \\
0 & 0 & \frac{1}{2} \Delta^{X} & E^{e^{h}}+p F
\end{array}\right)
$$

The Hamiltonian for the biexciton singlets $\left(\begin{array}{l}20 \\ 20\end{array}\right),\left(\begin{array}{l}20 \\ 11\end{array}\right)_{S}$ or $\left(\begin{array}{l}11 \\ 20\end{array}\right)_{S},\left(\begin{array}{l}20 \\ 02\end{array}\right)$ or $\left(\begin{array}{l}02 \\ 20\end{array}\right)$, and one of the three degenerate and fully decoupled triplets $\left(\begin{array}{l}20 \\ 11\end{array}\right)_{T}$ or $\left(\begin{array}{l}11 \\ 20\end{array}\right)_{T}$ of the biexciton is

$\widehat{H}_{X X}=\widehat{I}_{\varepsilon_{X X}}+\left(\begin{array}{cccc}0 & \frac{\sqrt{2}}{2} \Delta^{X} & 0 & 0 \\ \frac{\sqrt{2}}{2} \Delta^{X} & E_{1}^{h(e)}+p F & \frac{\sqrt{2}}{2} \Delta^{X} & 0 \\ 0 & \frac{\sqrt{2}}{2} \Delta^{X} & E_{2}^{h(e)}+2 p F & 0 \\ 0 & 0 & 0 & E_{1}^{h(e)}+p F\end{array}\right)$

Here $\varepsilon_{X(X X)}$ is the confinement energy of an exciton(biexciton) in a single QD (here bottom QD), $\widehat{I}$ is an identity matrix and $E^{e h}, E_{1}^{h(e)}$ and $E_{2}^{h(e)}$ are the Coulomb energies that are required to move one or two holes(electrons) from the bottom to the top QD.

[14] Values used for Fig. 2(a): $\Delta^{X}=0.45 \mathrm{meV}, J^{\text {eh }}=0.20$ $\mathrm{meV}, E^{e h}=17.6 \mathrm{meV}, E_{1}^{h}=14.87 \mathrm{meV}, E_{2}^{h}=70.4$ $\mathrm{meV}\left(4 E^{e h}\right)$ and $p=-1.02 \mathrm{meV} /(\mathrm{kV} / \mathrm{cm})$.

[15] Values used for Fig. [5(c): $\Delta^{X}=1.65 \mathrm{meV}, J^{\text {eh }}=0.20$ $\mathrm{meV}, E^{e h}=18.6 \mathrm{meV}, E_{1}^{e}=24.94 \mathrm{meV}, E_{2}^{e}=74.4 \mathrm{meV}$ $\left(4 E^{e h}\right)$ and $p=1.29 \mathrm{meV} /(\mathrm{kV} / \mathrm{cm})$. 